\title{
DINAMICA INDUSTRIAL DE OURENSE EN EL SIGLO XX
}

\author{
José SOMOZA MEDINA
}

Departamento de Geografía. Universidad de León

Ourense es a comienzos del siglo XXI una ciudad media caracterizada desde el punto de vista funcional por su oferta de bienes y servicios a una población provincial en continuo descenso. No obstante, la importancia relativa de su sector fabril la sitúa como tercer polo industrial de Galicia, tras Vigo y A Coruña, con una estructura diversificada donde sobresalen las empresas de tamaño medio. La evolución de esta actividad durante el siglo XX responde perfectamente al modelo de industrialización de las ciudades medias españolas, de tal forma que su estudio pormenorizado puede ayudarnos a establecer las principales pautas del lento y difícil camino de la industria en nuestro país. Por tanto, en este trabajo se pretende dar a conocer la dinámica industrial de Ourense en la pasada centuria, remarcando los distintos períodos evolutivos que configuran el paso de una primera etapa premaquinista al desarrollo tecnológico actual, como ejemplo práctico del modelo de industrialización de estas ciudades en España.

\section{LA ACTIVIDAD INDUSTRIAL EN 1900.}

Según el Censo de 1900 Ourense contaba con 15.194 habitantes, de los cuales tan sólo 1.536 personas, el $25,8 \%$ de la población activa, se dedicaban al sector secundario. La mayor parte de estos trabajadores se encuadraban bajo el epígrafe 4 del Censo -industrias clasificadas por las necesidades a que se aplican- que incluía talleres de confección, muebles, materiales de construcción, alimentación y auxiliar del transporte (Beiras Torrado, 1970). Ya en aquel momento el terciario agrupaba a un mayor porcentaje de la población activa, $38,7 \%$, seguido del primario, con un $35^{\prime} 5 \%$.

El listado de Contribución Industrial del Ayuntamiento de Ourense ofrece una información más detallada de las fábricas existentes en aquel momento. La Tarifa $3^{\mathrm{a}}$, en las que se censaban las máquinas de producción industrial, recogía en el año económico 1899-1900 únicamente 21 licencias, el 4,1\% del total, con una carga fiscal de $14.676,31$ pesetas, lo que suponía el $16,9 \%$ del conjunto del impuesto frente al $55^{\prime} 9 \%$ que aportaba el comercio o el $27,2 \%$ de los servicios. Estas licencias se correspondían con una fundición de hierro, cinco imprentas, una fábrica de teja y ladrillo, siete molinos, dos fábricas de gaseosas, una central de energía eléctrica y cuatro "piedras de chocolate a brazo". Analizando su estructura nos encontramos, por una parte, con industrias tradicionales, como los talleres artesanales de chocolate todavía movidos por la fuerza humana o los molinos harineros asentados en los cursos fluviales, pero también aparecen elementos 
nuevos que reflejan el carácter urbano del núcleo, como las fábricas de gaseosas, las imprentas o la central eléctrica. La fábrica de teja y ladrillo responde a la necesidad impuesta por la paulatina renovación del caserío y la edificación de nuevas viviendas, si bien el subsector de la construcción apenas reviste importancia en esta etapa. La única industria que verdaderamente puede recibir tal nombre a comienzos de siglo era la Fundición Malingre, creada por el belga Manuel Malingre Parmentier en 1849. Curiosamente, la llegada de este joven empresario a Ourense a medidos del siglo XIX es contemporánea al establecimiento de otras dos importantes fundiciones creadas por dos compatriotas suyos en A Coruña (Wonembuger) y Vigo (Sanjurjo).

No es este el único ejemplo de industrial de origen foráneo durante los primeros años del siglo XX. La fábrica de teja y ladrillo, ubicada como la Fundición en el barrio de O Couto, había sido fundada por el mallorquín Jaime Borrás Lladó, Otto Bodmer, de origen suizo, estableció un taller de relojería, Juan Iglesias Malleira, portugués, una sombrerería, además de otros industriales como Pedro Martínez Fernández de Jaén, o Andrés Perille de A Coruña. Lo mismo ocurría en los otros sectores económicos con banqueros riojanos y castellanos, ferreteros asturianos y vascos, comerciantes de tejidos de Valladolid, Barcelona, Zamora o Logroño, almacenistas de alimentación de Valladolid y Zamora, abogados de Madrid, Barcelona o Salamanca, médicos de Valladolid, Santander o Zaragoza, etc., etc.

Esta afluencia de empresarios y profesionales foráneos está íntimamente relacionado con el desarrollo de las comunicaciones y el hecho de ser Ourense capital provincial. Algunos arrieros que tenían este núcleo como uno de sus destinos se establecieron definitivamente en él, transformándose como consecuencia de la mejora de los transportes y las vías de comunicación en almacenistas y comerciantes. Otros vieron en la ciudad buenas perspectivas económicas y llegaron a través de estas nuevas vías para constituir sus negocios. Además junto a los titulares de los establecimientos llegaron familiares, amigos y dependientes que poblaron la ciudad gallega de naturales de Logroño, Zamora, Valladolid o Asturias. Su éxito animó a algunos vecinos a recalar en Ourense en años posteriores, conformándose de este modo una nutrida red de industriales, banqueros y comerciantes foráneos. Los agentes de esta "colonización comercial" (Otero Pedrayo, 1973) fueron quienes dirigieron el desarrollo experimentado por la ciudad en la segunda mitad del siglo XIX y comienzos del XX, muchas veces desde el puesto de alcalde, y el origen del poder que el comercio, y algunas familias en particular, detentaron desde entonces.

En resumen, la estructura industrial de Ourense a principios del siglo XX se basaba en tres subsectores: alimentación, metal-mecánica y auxiliar de la construcción, con un reducido porcentaje de propietarios de origen local. La industria alimentaria estaba representada por los molinos harineros, principalmente de maíz y centeno, las fábricas de chocolate y las que producían hielo y gaseosas. El chocolate era un producto muy apreciado por la burguesía urbana y tenía además un amplio mercado provincial, llegando en 1908 a contabilizarse hasta 13 fábricas de chocolate. Las fábricas de hielo y gaseosas se relacionaban con los cafés de la ciudad, lugares de gran importancia en la vida social de estos años. El subsector metalmecánico se originó a partir de la producción de hierro de la fundición de 
Malingre Parmentier. Esta fábrica, con sus cadenas de producción, relaciones patronal-empleados, estructura de ventas y marketing, fue el mejor ejemplo, sino el único, de empresa industrial en Ourense durante muchos años. Por último, la fabricación de materiales de construcción se desarrollaba en carpinterías industriales, aserraderos y hornos de cerámica, sin olvidar que muchas de las piezas que se fundían en la fábrica del Couto tenían como destino final formar parte de las viviendas del casco urbano (balcones, vigas, escaleras, verjas, cocinas, canalizaciones, columnas, puertas, etc.).

\section{LA SITUACIÓN EN 1930.}

La población en 1930 ascendía a 21.579 habitantes. En el sector secundario se agrupaba el $25 \%$ de la población activa, 2.353 personas, por encima del porcentaje que mostraba el primario, $18,7 \%$, pero a una gran distancia del terciario, $56,3 \%$. La confección era la actividad que empleaba a un mayor número de trabajadores, 536, de los cuales 251 eran mujeres, seguido de la industria metalmecánica, 265, la industria de la madera, 264, el subsector de la construcción, 237, y la industria alimentaria, 82 .

Desde comienzos del siglo, el sector fabril experimentó cierto crecimiento, multiplicándose el número de pequeños talleres metal mecánicos y auxiliares de la construcción, pero la ciudad seguía careciendo de factorías de tamaño medio o grande.

En 1916 se registraban 36 licencias industriales, en 1930, 158. El salto cuantitativo es importante, pero en la estructura del sector se aprecian pocos cambios. No existe, en realidad, una diversificación de la producción fabril. Las ramas específicas predominantes en la etapa anterior continúan siendo las mismas en ésta: auxiliar de la construcción, agroalimentaria y metal-mecánica. En comparación con la estructura del período anterior se debe destacar la mayor presencia del sector relacionado con la construcción frente a los otros dos, fruto de la expansión edificativa y de la importancia que este subsector comienza a adquirir en la ciudad.

TABla 1. Matrículas de la Tarifa 3 en la Contribución Industrial de 1930.

\begin{tabular}{llr}
\hline Tarifa 3. & \multicolumn{1}{c}{ Tipo de industria } & Número \\
\hline${\text { Clase } 1^{\mathrm{a}}}^{\mathrm{a}}$ & Carda mecánica & 1 \\
Clase $3^{\mathrm{a}}$ & Taller cerrajería y metálico & 24 \\
Clase $4^{\mathrm{o}}$ & Cepilladoras, taladradoras, molduradoras, sierras taller & 69 \\
\hline Clase $5^{\mathrm{a}}$ & Laboratorios farmacia y análisis químicos & 5 \\
Clase $6^{\mathrm{o}}$ & Fábrica de lejía & 1 \\
Clase $7^{\mathrm{a}}$ & Hornos y fábricas de biselar & 4 \\
\hline Clase $9^{\mathrm{a}}$ & Fábricas de caramelos, chocolate, gaseosas, licores, molinos, etc. & 39 \\
Clase $10^{\mathrm{a}}$ & Imprentas, máquinas de prensa & 13 \\
Clase $12^{\mathrm{a}}$ & Fábricas de hielo y máquinas de pulir piedra & 2 \\
\hline Total & & 158
\end{tabular}

FUENTE: VALCÁRCEL LóPEZ, 1992 
La Tarifa 3 únicamente representaba este año el 12,02\% de las cuotas totales y el $17,65 \%$ de las matrículas censadas. Las 158 licencias citadas, presentaban una cuota final de $41.552,40$ pesetas, siendo el total de 895 licencias y $345.564,67$ pesetas. La preponderancia del sector terciario, representado por las otras tres Tarifas, es evidente.

En cuanto a las empresas, en la industria agroalimentaria destacaban las fábricas de chocolate de Julio Domínguez (Chocolates Burgas y Nuestra Señora de los Remedios), Felipe Lorenzo Feijoo (Chocolates Chaparro) y María Blanco Portugal, las de licores de Fermín Fernández, Patricio Martín Sánchez (Ponches La Gallega) y Eloy Viso (Licores Viso), y el tostadero de café de Santiago Campos Ramos (Cafés Campos), todos ellos naturales de Ourense. La mayor parte de estas fábricas se localizaban entre las calles de Santo Domingo y Progreso, ubicadas en el sector central de la ciudad, con establecimientos de venta directa al consumidor.

En la industria auxiliar de la construcción sobresalían los talleres de otros empresarios ourensanos como Secundino Couto Solla, Benito Canal Yela, Luis Nóvoa o Eugenio Lorenzo Vidal y los almacenes de materiales de construcción de Estanislao Reverter, Felipe Castro y Juan Villar.

Tras el fallecimiento del fundador Manuel Malingre Parmentier en 1916, los talleres metálicos y la fábrica de fundición se repartieron entre sus hijos Antonio y Manuel Malingre Ludeña. Esta empresa tradicional influyó de manera determinante en la creación de otros talleres metal-mecánicos como los de Rogelio Fernández, los Hermanos Fraga o Severino Fernández.

Dos nuevos sectores comienzan a tomar relevancia en esta etapa, automoción y materiales eléctricos. Los talleres de automóviles se sitúan preferentemente en las carreteras de acceso a la ciudad y están asociados generalmente a concesionarios de alguna marca concreta (Citröen, Chevrolet, Chrysler, Hispano-Suiza). En cuanto al segundo sector, destacaban los talleres de Antonio Aragonés y David Ferrer Quirós, ambos ubicados en la céntrica calle del Progreso.

Por su parte, el subsector de la construcción inició en esta etapa un rápido crecimiento. En 1920 se censaron 1.000 edificios de viviendas en el núcleo urbano de Ourense, en 1940 eran 1.303. En esas dos décadas el incremento fue del $30 \%$, frente a la mínima fluctuación experimentada en el mercado de viviendas en los primeros veinte años del siglo. Además, las mejoras urbanas que demandaban los vecinos, como asfaltado de calles, enlosado de aceras, canalizaciones de aguas y alcantarillado, también comenzaron a generalizarse durante esta época, garantizando a los empresarios de la construcción la continuidad de la actividad y a los obreros la posibilidad de sustento. No es de extrañar entonces que empresarios industriales y grandes comerciantes canalizaran parte de sus activos hacia la construcción, un negocio que generaba elevadas rentabilidades.

Por tanto, en esta etapa se multiplican las pequeñas empresas industriales a manos de propietarios locales o descendientes de los fundadores de origen foráneo. Unos talleres y fábricas que se sitúan por lo general en espacios céntricos para impulsar su función auxiliar de centros de venta de los productos finales, destinados a una demanda provincial y fundamentalmente doméstica. El subsector de la construcción comienza a desarrollarse como la industria principal de la ciudad. 


\section{POSGUERRA E INDUSTRIAS DE SUSTITUCIÓN DE IMPORTA- CIONES.}

Los primeros años de la dictadura de Franco se caracterizan desde el punto de vista económico por la penuria generalizada de la posguerra y la instauración de un régimen basado en la autarquía, con la que se pretende sacar al país de esa grave situación. El cierre de los mercados exteriores obliga a crear una estructura industrial basada en la sustitución de importaciones, con lo que se crean numerosas empresas de tamaño medio que pretenden abastecer al mercado nacional de aquellos productos que antes llegaban de otros países.

En 1930, el porcentaje de la población activa del municipio de Ourense que se dedicaba al sector primario era el $18,7 \%$, diez años más tarde se situaba en el $28 \%$. Esta rerruralización se produce como consecuencia de la crisis bélica, en un contexto en el que el campo se convierte en colchón económico donde poder resguardarse. En 1940 la proporción de empleados en el sector secundario había descendido desde el $25 \%$ del censo anterior hasta el 18,5\%, para incrementarse de forma acelerada en 1950 hasta el 30'9\%, agrupando ahora a 6.833 personas sobre una población total de 55.574 habitantes, si bien 2.991 personas pertenecían al subsector de la construcción.

En esta etapa, la industria ourensana estaba estructurada básicamente en cinco sectores: madera, alimentación, metalurgia, automoción y auxiliar de la construcción, con un desarrollo algo menor de otras ramas como el vestido o la industria química. La TABLA 2, en la que aparecen las licencias de talleres industriales del censo de 1955 agrupadas por sectores de actividad, permiten confirmar esta estructura.

TABLA 2. Licencias industriales por ramas de actividad en 1955.

\begin{tabular}{lr}
\hline \multicolumn{1}{c}{ Actividad } & Número \\
\hline Madera & 165 \\
Alimentación & 150 \\
Metal-mecánico & 76 \\
Automoción & 48 \\
\hline Auxiliar de construcción & 42 \\
Vestido y confección & 30 \\
Otros & 89 \\
\hline Total & 600 \\
\hline FUENTE: INE, 1956
\end{tabular}

Estos talleres se localizaban generalmente en la periferia inmediata a la ciudad, cerca de las vías de acceso, como la carretera de Reza, Avenida de Zamora, carretera de circunvalación, Avenida de Portugal, Marcelo Macías, Capitán Cortés, Avenida de Buenos Aires, Ervedelo o carretera de Santiago, aunque también se podían encontrar establecimientos de este tipo en el casco histórico o en las calles del centro, y especialmente en la del Progreso. El barrio de A Ponte era el lugar donde existía una mayor concentración de naves industriales, seguido del Couto y 
la Avenida de Buenos Aires, pero sin que existiera ninguna zonificación planificada.

El mejor ejemplo de estos años, en los que la industria se dedicaba a sustituir los productos de exportación, se personaliza en Ourense en la figura del industrial Eduardo Barreiros, nacido en 1919, y que comenzó entre 1949 y 1953 a transformar motores de gasolina en diesel, en su fábrica instalada en el barrio de As Lagoas. En 1954 se trasladó a Madrid donde fundó la empresa Barreiros Diesel S.A. dedicada a la fabricación y comercialización de motores y vehículos. En Ourense creó con créditos de la Caja de Ahorros la empresa Barreiros Orense, donde se elaboraban piezas de automoción y armamento, que pasaría años después a denominarse Forjas de Galicia S.A. Eduardo Barreiros fue considerado durante los años cincuenta y sesenta como el principal empresario del motor en España, y sirvió de ejemplo entre los industriales locales, que impulsaron a la sombra del éxito alcanzado por este emprendedor hombre de negocios la modernización de sus instalaciones fabriles.

\section{POLÍTICA ESTATAL Y POLÍGONOS INDUSTRIALES.}

Con una coyuntura internacional favorable, la puesta en marcha del Plan de Estabilización Nacional de 1959 produjo en la economía española un fuerte desarrollo. Dentro de la estrategia orquestada por la tecnocracia gobernante se encontraba la política de polos industriales, mediante la cual se promovió con grandes subvenciones públicas la industrialización de una serie de enclaves distribuidos por el territorio nacional y alejados de los principales centros. Los polígonos industriales comenzaron a situarse entonces en las ciudades medias españolas, en una dinámica que terminó extendiéndose por todo el país. Toda ciudad quería tener su polígono, como símbolo de los nuevos tiempos que se avecinaban y garantía de éxito económico. En este contexto las facilidades para la emigración, el progresivo aumento del nivel de vida de los españoles y la promoción turística del país, conseguían mantener ritmos de crecimiento muy elevados. La entrada de capitales se producía por la masiva afluencia de turistas y el envío de las remesas de los emigrantes, mientras el mercado interno se fortalecía con el lento aumento de los salarios, que derivaba a su vez en un incremento considerable del consumo.

En 1970, el 30,9\% de la población activa se dedicaba al sector secundario, y concretamente el $16,8 \%$ a la industria, cuando el terciario agrupaba ya al $61,5 \%$ del total.

En este período surgen numerosas iniciativas empresariales ligadas al sector secundario. La economía urbana, profundamente influida por la emigración, asiste al desarrollo de sus factores productivos, que sitúan a Ourense desde ese momento en el tercer lugar en la escala de desarrollo industrial de Galicia. Aumenta el capital circulante con las remesas depositadas en las entidades de crédito, y fundamentalmente en la Caja de Ahorros Provincial, que se dirigen de forma preferente hacia el mercado de la vivienda pero alcanzan también para financiar la inversión industrial. Asimismo se incrementan y desarrollan los recursos humanos, por un lado la mano de obra aumenta con el crecimiento demográfico de la ciudad, al mismo tiempo que crece la clase empresarial, con nuevos proyectos 
que tratan de seguir la estela de éxito marcada por los grandes personajes industriales de Ourense. A menudo estas nuevas empresas están dirigidas por antiguos emigrantes que desarrollan en la ciudad los conocimientos técnicos adquiridos en sus puestos de trabajo en Europa, con lo que el factor tecnológico o "KnowHow" también forma parte integrante de este desarrollo. Entre los factores externos sobresale el crecimiento cuantitativo y cualitativo del mercado urbano y los incentivos emanados de la Ley de creación de la Gran Área de Expansión Industrial de Galicia, que suponen unas ventajas comparativas que no existían en etapas anteriores. Por último, la oferta de suelo industrial concretada en el polígono de San Cibrao das Viñas completa el elenco de condicionantes positivos que influyen en el desarrollo del sector secundario en este período.

La ubicación de los talleres industriales se encontraba en los años 1960 repartida por distintos lugares de la periferia urbana -Veintiuno, Avenida de Zamora, Avenida de Portugal, Couto, Puente, Lagoas- hasta que la creación del polígono y las ayudas ofrecidas por la Caja de Ahorros Provincial para financiar su traslado, agruparon las distintas naves en el espacio de más trascendencia para el desarrollo industrial de Ourense.

En mayo de 1961 el Ayuntamiento solicitó a la Gerencia de Urbanismo el establecimiento dentro del término municipal de un polígono. La petición no tuvo una respuesta positiva y en 1966 las instituciones oficiales ourensanas; Ayuntamiento, Diputación y Gobierno Civil, junto con la Caja de Ahorros Provincial realizaron las gestiones oportunas para crear uno de forma independiente. En total 6.000 parcelas agrícolas, que suponían casi 74 hectáreas de extensión, se transformaron en suelo industrial en el vecino municipio de San Cibrao das Viñas. Para facilitar el desarrollo de este parque industrial, en principio propiedad de la $\mathrm{Ca}$ ja de Ahorros, los agentes locales contactaron con el empresario Eduardo Barreiros, quien finalmente estableció en el espacio urbanizado la filial Barreiros Orense. La iniciativa supuso para la entidad de crédito una inversión de 600 millones de pesetas, contabilizándose a finales de los años 197030 empresas instaladas en el polígono.

El parque pasó a ser oficialmente polígono industrial en virtud del Real Decreto 1980 de 31 de marzo de 1974, siendo incluido dentro de la Gran Área de Expansión Industrial de Galicia (GAEIG) el 5 de julio de ese mismo año. A partir de entonces Sigalsa, sociedad de creación de suelo industrial de la GAEIG y de la Sociedad de Desarrollo Regional, fue la encargada de financiar las siguientes ampliaciones. La primera de ellas, realizada en 1975, supuso la adición de 56 hectáreas, siendo la mayor parte de ese espacio ocupado por la fábrica que Citröen comenzó a instalar en 1977, aunque sus naves no iniciarían la producción de núcleos de ruedas, cárteres de caja de velocidades y líneas de estribos de frenos hasta 1979. Más tarde vendrían nuevas ampliaciones hasta llegar a las 291 hectáreas con las que cuenta actualmente el polígono.

En la distribución del empleo industrial en 1970 por ramas de actividad (TABLA 3), se aprecia como los subsectores tradicionales continúan caracterizando la estructura fabril de Ourense. Cada uno de ellos con unas circunstancias específicas que marcan su propia evolución:

- Madera: Con un claro desarrollo tanto en la rama del mueble como en la auxiliar de la construcción. Subsector que contaba con algunas empresas 
de tamaño medio, además de numerosas carpinterías y mueblerías de menores dimensiones.

- Alimentación: Subsector tradicional que experimenta en este período un fuerte crecimiento gracias a la expansión de las Cooperativas Orensanas (Coren), sociedad cooperativa de transformación de los productos agropecuarios fundada por Eulogio Gómez Franqueira.

\section{Figura 1. Polígono industrial de San Cibrao das Viñas.}

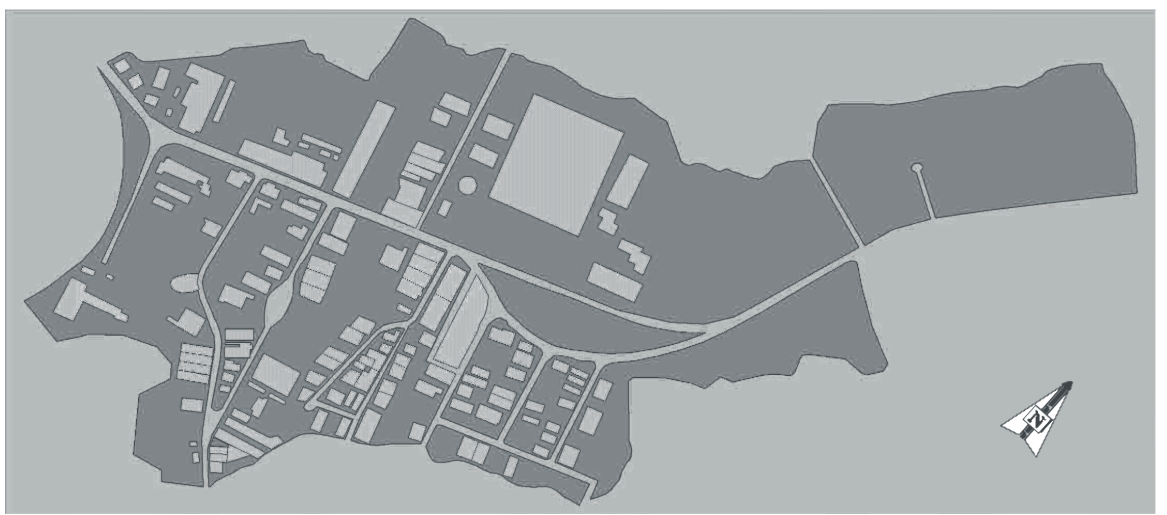

(FUENTE: www.igatel.es)

- Metalurgia: Junto a las cuatro fundiciones existentes en este período aparecen pequeños establecimientos de soldaduras y fabricación de estructuras metálicas, directamente relacionados con otros dos subsectores, el auxiliar de la construcción y el de automoción.

- Automoción: Concentración empresarial y elevado número de trabajadores en los establecimientos principales, sobresaliendo dos empresas de carrocerías de autobuses, una de furgones isotermos y tres talleres de reparación.

- Confección: Dispersión de la actividad en un gran número de talleres de confección, calzado, piel y dos fábricas textiles. Subsector que daba empleo a un elevado porcentaje de la población asalariada industrial de $\mathrm{Ou}-$ rense, con una importante presencia del trabajo a domicilio.

- Auxiliar de la construcción: Integrado por carpinterías de madera y metálicas, explotaciones de áridos, canteras y fabricación de materiales de construcción. Subsector que pierde importancia con respecto a etapas anteriores frente al desarrollo de otras ramas fabriles.

El Censo Industrial de 1978 contabilizaba 337 establecimientos en la capital provincial, a los que habría que añadir los talleres existentes en la periferia urbana y especialmente en el Polígono. La TABLA 4 muestra, una vez más, la preponderancia de los sectores tradicionales; madera, alimentación, metal-mecánica, auxi- 
liar de la construcción y textil.

TABLA 3. Distribución del empleo industrial por ramas de actividad en 1970.

\begin{tabular}{lrr}
\hline Actividad & Empleos & \% \\
\hline Textil, confección y piel & 827 & 23,24 \\
Alimentación y bebidas & 689 & 19,36 \\
Madera y mueble & 673 & 18,91 \\
Metalurgia de transformación y automoción & 624 & 17,54 \\
Auxiliar de la construcción & 183 & 5,14 \\
Papel y artes gráficas & 172 & 4,83 \\
Metálicas básicas & 168 & 4,72 \\
Química y derivados & 163 & 4,58 \\
Otras industrias manufactureras & 60 & 1,68 \\
\hline Total & 3.559 & 100 \\
\hline
\end{tabular}

FUENTE: PRECEDO LEDO, 1991.

TABLA 4. Establecimientos fabriles en Ourense según el Censo Industrial de 1978.

Clase de Actividad

Captación, depuración y distribución de agua

Total

Industrias de productos minerales no metálicos ni energéticos 31

Industria química

Fabricación de productos metálicos

Construcción de maquinaria y equipo mecánico

Construcción de maquinas de oficina y material eléctrico

Construcción de vehículos y otro material de transporte

Fabricación de instrumentos de precisión

Industrias de productos alimenticios, bebidas y tabacos 77

Industria textil y del cuero

Industria del calzado, vestido y otras confecciones textiles 33

Industria de la madera, corcho y muebles de madera 98

Industria del papel

Industrias de transformación del caucho y materias plásticas $\quad 7$

Otras industrias manufactureras

FUENTE: INE, 1979

En este período se multiplica el número de establecimientos industriales y se diversifica la oferta de productos, aunque en su estructura predominan los sectores tradicionales. La promoción del polígono industrial y la presencia de capitales procedentes de la emigración son los factores fundamentales de este desarrollo, que se verá incrementado en la última fase. En cuanto a la evolución particular de algunas empresas significativas cabe señalar el cierre en 1979 de la Fundición Malingre tras el fallecimiento de Manuel Malingre Lamas, nieto del fundador, que dirigía desde 1930 la que había sido primera factoría industrial de Ourense. 


\section{EL PARQUE TECNOLÓGICO Y LA DISPERSIÓN DE LA ACTIVI- DAD INDUSTRIAL.}

La actividad fabril en Ourense ha experimentado en los últimos años del siglo $\mathrm{XX}$ un leve desarrollo general en medio de una profunda transformación de su estructura. Los efectos de la crisis energética de 1973 se hicieron sentir en la ciudad especialmente tarde, pues no sería hasta principios de los años ochenta cuando comenzó a generalizarse el cierre de numerosas empresas industriales, incluido el desmantelamiento en 1984 de la planta de Citröen en el polígono de San Cibrao das Viñas. Entre 1970 y 1981 el empleo industrial experimentó un fuerte crecimiento, coetáneo al desarrollo del polígono, de tal forma que de los 4.070 activos de 1970 se pasó a los 6.168 de 1981 (51,54\% de incremento). En la década siguiente, la población encuadrada en la industria apenas sufrió modificaciones en su volumen global, 6.143 ocupados, para mostrar en el padrón de 1996 un descenso importante, hasta contabilizar 5.595 empleos (-9\% en cinco años), lo que significaba el $16,9 \%$ de la población activa. Esta irregular evolución enmascara el fin de un proceso de industrialización y el comienzo de un nuevo modelo económico.

El fracaso de las industrias de tamaño medio-grande en Ourense, incapaces de competir en el mercado internacional por su escaso desarrollo tecnológico, supuso numerosos expedientes de regulación de empleo y en muchos casos desaparición de empresas, mientras las industrias de menor tamaño, más flexibles, lograban afianzarse y crecer en número. En otras palabras, se produjo una clara sustitución del factor trabajo por una mayor inversión en tecnología y un redimensionamiento de las unidades de producción de acuerdo con la estructura económica general de Galicia. A este proceso de adaptación a unas nuevas condiciones le acompañó otro de recolocación sobre el espacio de las naves industriales, abandonando el área central para ubicarse en su periferia. Dentro de la apuesta institucional por superar la crisis y adaptarse a los nuevos tiempos se sitúa la ubicación del Parque Tecnológico de Galicia en San Cibrao das Viñas y no en la periferia industrial de los dos núcleos más dinámicos de la región, tal y como habían aconsejado los informes previos.

La única empresa de grandes dimensiones en Ourense a finales del siglo XX es Coren, líder en el sector alimentario gallego junto con la viguesa Pescanova y con una facturación en el año 2000 que superaba los cien mil millones de pesetas. Este éxito se debe a la continua adecuación de sus estructuras productivas, financieras y comerciales a las exigencias del mercado, dentro de un proceso de integración vertical y horizontal que crea una verdadera agricultura de grupo. Desde la fabricación de piensos, importando las materias primas de los cinco continentes, hasta la comercialización de los productos que ofrecen las distintas cooperativas en países como Rusia, Uruguay o Rumania, todas las fases se dirigen desde la propia empresa.

Algunas de las instalaciones de Coren se encuentran en el Polígono Industrial de San Cibrao das Viñas, que presenta en este período una profunda transformación en el tamaño medio de sus empresas. En la TABLA 5 se aprecia como el número de establecimientos se duplica, aunque la población empleada apenas varía. Este hecho confirma el redimensionamiento de las empresas industriales que 
invierten en tecnología y reducen las plantillas de empleados. Por otro lado, en la tabla también se observa el mayor peso en la estructura del polígono de las empresas dedicadas a las construcciones metálicas, muebles, química del plástico, alimentación, materiales de construcción y auxiliar del automóvil. Respecto a etapas anteriores sólo se constata la aparición de una rama fabril nueva, como es la de los materiales plásticos, de gran importancia en la producción industrial más reciente, frente a la perdurabilidad de los otros sectores de actividad, prácticamente los mismos que caracterizaban a la industria ourensana a comienzos del siglo XX.

TABLA 5. Empresas industriales en el polígono de San Cibrao das Viñas.

\begin{tabular}{lrr}
\hline Sectores de Actividad & $\mathbf{1 9 8 5}$ & $\mathbf{2 0 0 0}$ \\
\hline Alimentación & 6 & 10 \\
Auxiliar del automóvil & 2 & 7 \\
Calzados & 2 & 1 \\
Colchones & 0 & 2 \\
Construcciones metálicas & 10 & 18 \\
Electricidad y electrónica & 2 & 6 \\
Energía & 0 & 2 \\
Materiales de construcción & 5 & 9 \\
Mueble y madera & 4 & 13 \\
Química (Plástico) & 6 & 13 \\
Textil y confección & 3 & 2 \\
Construcción & 1 & 1 \\
\hline Total & 41 & 84 \\
\hline
\end{tabular}

FUENTE: PRECEDO LEDO, 1991 y www.xanela.com

En el ámbito municipal la crisis afectó sobre todo a las pequeñas industrias de base familiar con escasa capitalización, cerrando numerosos talleres tanto metalmecánicos como de transformación de minerales no metálicos, del sector alimentario, textil o de la madera. En el Censo de Locales de 1980 se registraban 514 establecimientos industriales y 3.209 personas empleadas, en el de 1990361 locales y 3.079 empleos. Las empresas industriales que superaron la crisis aumentaron sus plantillas para cubrir la cuota de mercado que dejaban libre los talleres familiares, de tal forma que el reajuste en Ourense supuso finalmente el mismo resultado que en el polígono, el fortalecimiento de las empresas de tamaño pequeño-medio, con una importante capitalización.

En la TABLA 6 aparecen reseñadas las industrias establecidas en la comarca ourensana y su relación con el total provincial según el Impuesto de Actividades Económicas de 1999. En este cuadro se destaca el aumento general de los establecimientos industriales, la clara concentración de algunas actividades y la importancia de la ubicación industrial en la periferia del municipio central, en relación a los datos que obteníamos en el Censo de Locales de 1990.

Este último hecho se enmarca dentro de un proceso de desindustrialización del núcleo urbano e incremento de los establecimientos fabriles en el espacio rural 
inmediato, común en las sociedades avanzadas (Precedo Ledo, 1996). La recolocación espacial de la industria obedece a diversos factores de atracción y repulsión que interrelacionados provocan el desplazamiento de estas actividades a espacios cada vez más alejados de las ciudades. En un principio, la industria se situaba en los núcleos urbanos para aprovechar la disponibilidad de mano de obra y unas mejores comunicaciones, con el paso del tiempo las externalidades positivas se transformaron en deseconomías de escala y las fábricas se situaron en los suburbios y polígonos industriales próximos. Finalmente, la crisis de los setenta y noventa favoreció en el mundo desarrollado un nuevo movimiento de expansión hacia espacios más rurales, donde el abaratamiento de los costes de producción permitía competir de forma ventajosa en el mercado global.

TABLA 6. Empresas industriales en la comarca de Ourense y porcentaje respecto al total provincial en 1999.

\begin{tabular}{lrr}
\hline \multicolumn{1}{c}{ Sectores de Actividad } & $\mathbf{N}^{\mathbf{0}}$ & \% Prov \\
\hline Energía y Agua & 5 & 55,55 \\
Industria Extractiva y Transf. de minerales no metálicos & 55 & 21,15 \\
Industria Química & 15 & 65,21 \\
Industria Metal-Mecánica & 219 & 47,92 \\
Industria Alimentaria, Bebidas y Tabacos & 160 & 27,68 \\
Industria Textil y del Cuero & 97 & 55,11 \\
Industria de la Madera y del Mueble & 220 & 40,44 \\
Industria del Papel y Artes Gráficas & 33 & 60,00 \\
Industria del Caucho y Materias Plásticas & 21 & 87,50 \\
\hline Total & 825 & 38,80 \\
\hline
\end{tabular}

FUENTE: www.ige.es

En el caso de Ourense estos factores se complementan con la permisividad de los primeros ayuntamientos democráticos que consintieron la aparición de naves industriales en las vías de acceso al término municipal y la decisión política de ampliar los espacios industriales de los municipios vecinos. Así, al Polígono Industrial de San Cibrao das Viñas y al Área Comercial Barreiros, se le han unido recientemente el Parque Tecnológico de Galicia y el Parque Empresarial de Pereiro de Aguiar, estando todavía en proyecto el Polígono Industrial de Barbadás ( $\mathrm{Fi}$ gura 2). La T ABLA 7 muestra el porcentaje de población activa comarcal ocupada en la industria y la distribución del número total de establecimientos. En ella se aprecia como únicamente el municipio capitalino reduce la proporción de activos, al tiempo que refuerza su pérdida de representatividad frente al importante crecimiento de los municipios situados al sur. 
FIGURA 2. Expansión industrial en la comarca de Ourense.

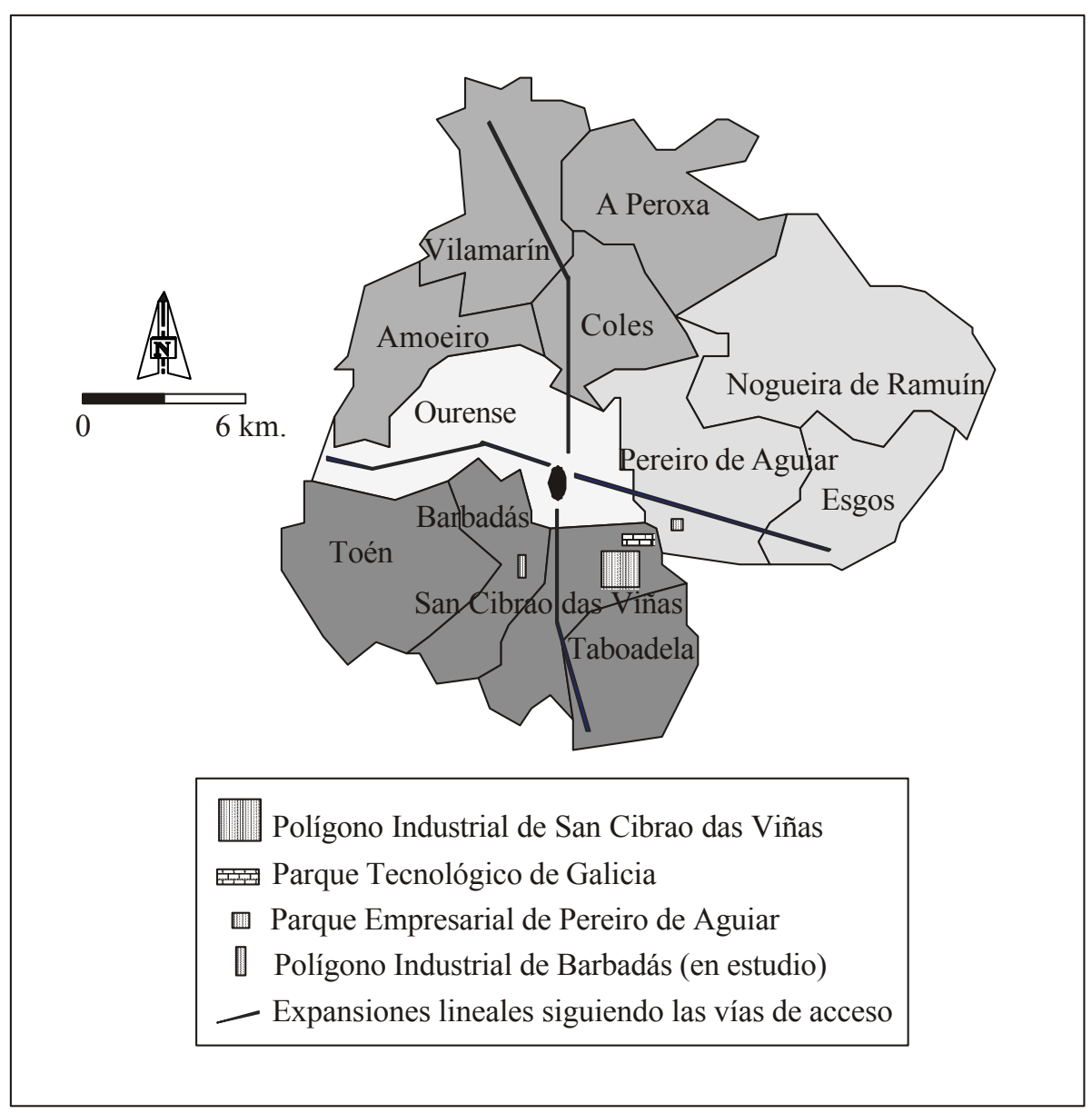

(FUENTE: LÓPEZ GONZÁLEZ, 2000)

TABLA 7. Distribución porcentual de la población comarcal ocupada en la industria y del número total de establecimientos.

\begin{tabular}{lcccr}
\hline & Pobl. ocup. en la industria & Establec. industriales \\
\hline & $\mathbf{1 9 8 1}$ & $\mathbf{1 9 9 6}$ & $\mathbf{1 9 8 0}$ & $\mathbf{1 9 9 8}$ \\
\hline Ourense & 19,10 & 16,83 & 61,6 & 51,9 \\
Periferia norte & 15,61 & 22,95 & 15,0 & 12,9 \\
Periferia este & 10,22 & 17,44 & 6,8 & 7,7 \\
Periferia sur & 14,36 & 23,07 & 16,6 & 27,5 \\
\hline
\end{tabular}

FUENTE: IGE, y LÓPEZ GONZÁLEZ, 2000 
En lo que respecta a las tipologías de la nuevas localizaciones industriales se puede distinguir entre las naves-escaparate que se distribuyen a lo largo de las vías de comunicación, los parques empresariales y la industrialización dispersa. Dentro del segundo modelo destaca el Parque Tecnológico de Galicia (PTG), inaugurado en 1992.

La creación del PTG supuso un cambio de gran importancia en el modelo de desarrollo del sector industrial, orientando la administración pública autonómica todos sus esfuerzos hacia el fomento de la investigación, la innovación y el desarrollo, con una inversión que superó los seis mil millones de pesetas. Dentro de la estructura de funcionamiento del Parque destaca el Centro de Empresas e Innovación (CEI), un edificio inteligente dotado de todos los servicios telemáticos que funciona como "incubadora" de proyectos empresariales.

En el diseño inicial se identificaron siete áreas de actividad sobre las que debía basarse el desarrollo del Parque Tecnológico, en relación a las potencialidades del espacio provincial: agroindustrial, pizarra y rocas ornamentales, forestal, textilconfección-moda, industria auxiliar del automóvil, materiales avanzados y medioambiente. La posterior evolución ha afianzado alguno de estos sectores, como el agroindustrial, textil, o materiales avanzados, y creado nuevas expectativas en otras ramas como la aeronáutica, diseño industrial, informática y telecomunicaciones.

\section{FIgURA 3. Plano del Parque Tecnológico de Galicia.}

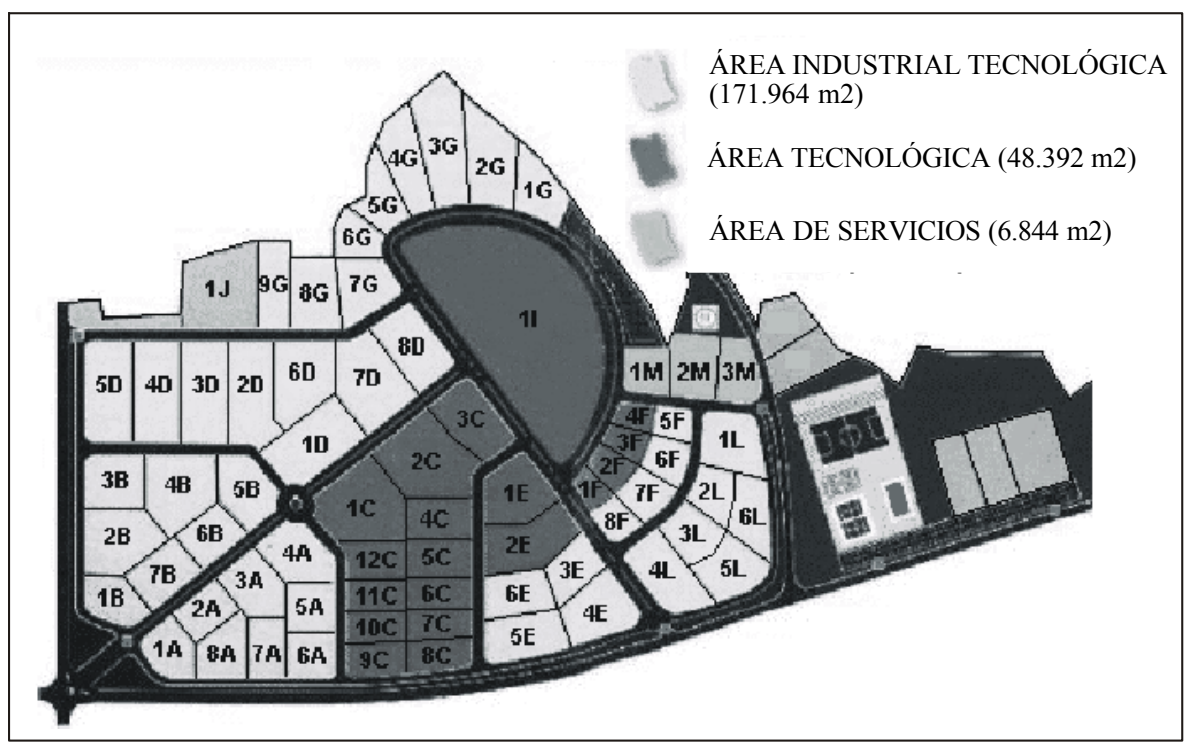

FUENTE: www.ptg.es

Si hace apenas unos años, en 1996, únicamente se encontraba ocupada una 
parcela, a finales del 2000 eran ya 30 las empresas asentadas en el Parque, a las que habría que añadir 5 Institutos públicos (Metrología, Centro Tecnológico de la Carne, Centro de Innovación y Servicios de la Madera, Sociedade Pública de Investimentos y Centro Teleservicio de Galicia). El futuro del PTG puede calificarse como esperanzador, aunque este desarrollo se siga beneficiando de las importantes subvenciones que aportan organismos públicos y privados desde la aprobación en 1996 del Plan de Relanzamiento del PTG, diseñado por la Xunta de Galicia.

\section{CONCLUSIONES.}

A lo largo de estas páginas hemos visto la dinámica industrial de Ourense en el siglo XX diferenciando cinco etapas. En la primera de ellas el escaso protagonismo industrial estaba representado por individuos emprendedores asentados en la ciudad tras el desarrollo de las comunicaciones y el incremento de las funciones que le correspondían como capital provincial. En una etapa posterior el impulso de estos pioneros incentivó el desarrollo de la clase empresarial local, aunque no se produjo un cambio estructural en el sector, sino más bien una continuación de los patrones marcados desde mediados del siglo XIX. A esta fase le sucedió en el tiempo el período marcado por la autarquía, con la consiguiente creación de empresas de sustitución de importaciones, que en el caso de Ourense se limitó a unos pocos ejemplos. Los años sesenta y setenta suponen la puesta en práctica de políticas nacionales de promoción del sector industrial, que tienen su repercusión en la creación y posterior desarrollo del polígono de San Cibrao das Viñas, donde la estructura de actividades muestra pocos cambios con respecto a etapas anteriores. Por último, las décadas finales del siglo se caracterizan por nuevas políticas de subvenciones, ahora de la administración autonómica, que crean el espacio de futuro del sector en Ourense, el Parque Tecnológico de Galicia. En este lugar y por primera vez se asiste a una modificación clara en la estructura de la actividad industrial, con la aparición de empresas aeronáuticas, de nuevos materiales, agroalimentaria, moda o telecomunicaciones con proyección internacional.

Hasta este último momento y exceptuando el breve intervalo productivo de Citröen, las industrias establecidas en Ourense se definían por sus reducidas dimensiones. Todavía hoy, la gran mayoría de los establecimientos industriales no cuentan con más de 50 empleados, una estructura adecuada para hacer frente a la demanda local o provincial pero insuficiente en un mercado más amplio y competitivo. Por otro lado, la dependencia de las subvenciones públicas afecta por igual a todas las empresas, que necesitan constantemente ayudas institucionales para asegurar su permanencia. De hecho, la falta de capitales en el sector industrial es uno de los principales problemas de esta actividad en Ourense y no como consecuencia de la inexistencia de éstos, sino de su canalización hacia otros sectores no productivos, representados principalmente por la construcción y especulación inmobiliaria.

En resumen, el lento y difícil camino de la industria en Ourense se caracteriza por la falta de una voluntad social emprendedora, que sólo parecen evidenciar algunos escasos ejemplos que sirven de excepción tras luchar contra la corriente, el apego atávico a los sectores tradicionales de actividad que se repiten incesantemente desde los tiempos premaquinistas $\mathrm{y}$, por último, la dependencia de las deci- 
siones políticas y los fondos públicos que impiden a los empresarios actuar con la libertad necesaria. No obstante, la creación y posterior evolución del Parque Tecnológico de Galicia puede ser un hito en la dinámica industrial de Ourense que imprima un nuevo rumbo a este sector. La respuesta sólo la puede dar el tiempo.

\section{BIBLIOGRAFÍA.}

- BEIRAS TORRADO, X. M. (1970) Estructura y problemas de la población gallega. A Coruña, Grafinsa

- INE (1956) Reseña estadística de la provincia de Ourense. Madrid

- INE (1979) Censo industrial de España. 1978. Madrid

- LÓPEZ GONZÁLEZ, A. (2000) «Evolución de la localización industrial en el área urbana de Ourense», en Industria y medio ambiente. Alicante, Universidad de Alicante, pp. 187-197

- OTERO PEDRAYO, R. (1973) Orense. Vigo, Bibliófilos Gallegos.

- Precedo Ledo, A. (1996) Ciudad y desarrollo urbano. Madrid, Síntesis.

- Precedo Ledo, A. (Dir) (1991) Ourense. Centro de equilibrio. Coruña, Fundación Caixa Galicia.

- Somoza Medina, J. (2001) Desarrollo urbano en Ourense. Tesis Doctoral inédita.

- Somoza MedinA, J. y LóPEZ GonZÁLEZ, A. (2001) “La ciudad de Ourense: la economía", en 100 años de historia con Ourense. Ourense, Cámara de Comercio de Ourense, pps. 95-131

- VALCÁRCEL LÓPEZ, M. (1992): Estructura económica e comportamentos políticos. Tesis Doctoral inédita.

RESUMEN: En este artículo se pretende mostrar la dinámica industrial de Ourense durante el siglo XX, caracterizando las diversas etapas que atraviesa este sector a lo largo del tiempo. Siguiendo la metodología del análisis inductivo, el estudio de un caso concreto nos puede aproximar al modelo general de industrialización de las ciudades medias españolas, de tal forma que las etapas identificadas en la evolución fabril de Ourense pueden, por extensión, ser perfectamente válidas para analizar otro caso concreto, como pueda ser el ejemplo de Toledo, Badajoz, Huelva o León. Estas etapas en las que se ha dividido la evolución industrial de Ourense son cinco: una primera caracterizada por la presencia de empresarios de origen foráneo, a la que sigue una fase de desarrollo de la clase empresarial local pero sin que se produzca un cambio estructural en el sector. La tercera etapa se corresponde con los años de la autarquía en los que el sector industrial se beneficia del proteccionismo estatal orientando su producción a la sustitución de importaciones. Las décadas posteriores se caracterizan por la política de polígonos industriales que lleva a cabo la administración tecnocrática, para concluir en la última etapa con las nuevas directrices emanadas de los gobiernos autonómicos, tendentes a potenciar la innovación y el desarrollo tecnológico como factores clave para superar la competencia en el mercado mundial que acompaña a la globalización de la economía.

$P A L A B R A S C L A V E$ : Industria, ciudades medias, Ourense, siglo XX

ABSTRACT: The main aim of this paper is to show the industrial dynamics of Ourense during 
the XX century, characterizing the diverse stages that it crosses along the time. Following the methodology of the inductive analysis, the study of a concrete case can approach to the general pattern of industrialization of medium-sized cities in Spain, in such a way that the stages identified in the industrial evolution of Ourense could, for extension, to be perfectly valid to analyze another concrete case, like the example of Toledo, Badajoz, Huelva or León. The stages in those the industrial evolution of Ourense has been divided are five: a first one characterized by the presence of managers of strange origin, to which follows a phase of development of the local managerial class but without a structural change in the sector. The third stage concerns with the years of the autarchy in those the industrial sector benefits of the state protectionism guiding its production to the substitution of imports. The later decades are characterized by the politics of industrial estates that carries out the technocratic administration, to conclude in the last stage with the new emanated guidelines of the autonomous governments, trying to promote the innovation and the technological development as key factors to overcome the competition in the world market that accompanies the globalization of the economy.

KEY WORDS: Industry, medium-sized cities, Ourense, XX century

$\boldsymbol{R} \boldsymbol{E} \boldsymbol{S U} \boldsymbol{M E}$ : Le but principal de cet article est de montrer la dynamique industrielle d'Ourense pendant le siècle XX, caractérisant les étapes diverses qu'elle croise le long du temps. Après la méthodologie de l'analyse inductive, l'étude d'un cas concret peut s'approcher a la configuration générale de l'industrialisation des villes moyennes en Espagne, de telle manière que les étapes identifiées dans l'évolution industrielle d'Ourense aient pu, pour l'extension, pour être parfaitement valides pour analyser un autre cas concret, comme elle peut être l'exemple de Toledo, Badajoz, Huelva ou León. Ces étapes dans ceux que l'évolution industrielle d'Ourense a été divisée sont cinq: un premier a caractérisé par la présence des gestionnaires d'origine étrange, auxquels suit une phase du développement de la classe gestionnaire locale mais sans changement structurel dans le secteur. La troisième étape appartient ainsi que les années de l'autarchie dans ceux les avantages industriels de secteur du protectionnisme d'état guidant sa production a la substitution des importations. Les décennies postérieures se charactérisent par la politique des polygones industriels qui effectue la gestion technocratique, pour conclure a la dernière étape avec les nouvelles directives émanées des gouvernements autonomes, essayant de favoriser l'innovation et le développement technologique pendant que les facteurs principaux pour surmonter la concurrence sur le marché mondial qui accompagne le globalization de l'économie.

MOTS-CLÉS: industrie, villes moyennes, Ourense, XX siècle 